\title{
The Social Layer of Stampers Ladder: A Systematic Approach to the Soft Edge of Organizational Transformations
}

\author{
Auke J.J. van Breemen ${ }^{1[0000-1111-2222-3333]}$ and Ralf Nieuwenhuijsen²[1111-2222-3333-4444] \\ ${ }^{1}$ KiF-advies, Oude Graafseweg 52, 6543PS Nijmegen, The Netherlands \\ info@KiF-advies.nl \\ ${ }^{2}$ Bibo Trainingen, Van Oldenbarneveltstr. 47, 6512 AT, Nijmegen \\ ralfebibotrainingen.nl
}

\begin{abstract}
The social layer of Stampers ladder addresses the problem of responsible agents interacting with each other. It is the layer at which in organizations decisions are made and transformations negotiated. The method we present supports this human interplay. It combines principles of actualism, ontology charts, the knowledge in Formation process model and the Cynefin framework to gather and combine quantitative data with qualitative data, expressing attitudes and perceptions in meaningful diagrammatic representations of business processes. The analytic tool Sensemaker can be used to support decision making.
\end{abstract}

Keywords: Actualism, Ontology Chart, Cynefin, Knowledge in Formation $(\mathrm{KiF})$, Stakeholder Focused Modelling, Process, Qualitative and Quantitative Data, Peircean Semiotics.

\section{$1 \quad$ Introduction}

If we, the developers, want to provide actionable insight into the human interplay among sign-based world and digital technologies as part of processes of organizational transformation, it is essential that they, the users, get insight in what is needed to realize the transformations and can share their experiences with their co-workers. This insight requires that each of the actors is afforded to have an idea of the situation (s)he is acting in and that those ideas are shared where needed. Here we face two alignment issues at once.

The first may be termed vertical alignment. Hoppenbrouwers directs the attention to the human side of information systems building with the distinction between business stakeholders and IT stakeholders. The former being primarily occupied with running the business, the latter with designing, building and maintaining information systems with the risk of misunderstanding and bad performance due to differing goals and conceptual habits in the design stage [1]. Stamper looks at it from a systems side when he proposes his semiotic ladder to distinguish the different levels that must be considered when designing an information system [2]. 
The second may be termed horizontal alignment. Its concern is interoperability across (departments of) organizations. Liu, Li and Liu following Stampers semiotic framework state that we have semiotic interoperability if signs among systems are successfully communicated in all the six levels Stamper distinguishes. Thus, every sign aspect is covered [3], [4]. Their interest concentrates on five of the six levels: physical world, empirics and syntactics, taken together under the label 'technical', semantic and pragmatic interoperability. This grouping relates their work to the Shannon Weaver distinction in three levels [5] of which the Stamper framework is a refinement from a semiotic perspective. The technical level being formally covered by Shannon's mathematical theory of communication. From there their research climbs the ladder layer by layer, leaving the social layer for the moment unexploited.

Goldkuhl and Ágerfalk remark that it is difficult to distinguish between the pragmatic and social levels on the semiotic ladder because in both signs are produced and such actions are in most cases social actions [6]. It appears that they forget that all levels of the ladder contribute in social actions. Stampers ladder levels are objectifications of sign aspects involved in signs: a word has its qualities (physical), its form and existence (empirics), its combinatory properties (syntactics), its meaning (semantics), its usability (pragmatics) and its habitat (social world). So, all levels of the ladder are involved in the social world, just as Shannon's theory on the technical level is involved in the meaning and effectiveness level. The crux being that it is possible to pay attention to the technical level without taking care of the other levels, but it is not possible, when designing information systems, to pay due attention to the social level without taking account of the involved levels, as the information revolution makes clear.

This leaves us with the question what, if any, the distinguishing characteristics are of the social level of Stampers ladder. Oppl and Hoppenbrouwers provide a good vantage point for an answer with their plea for stakeholder-centric modelling. In [7] they assert that involving business stakeholders in enterprise modelling also helps them to articulate and align their views on their organization. This requires, so they state, that stakeholders must be able to understand and perform conceptual modelling for representing their views on enterprise structure and behavior. I regard this to be part of the vertical view on alignment due to its reliance on the semantical layer and its modelling techniques. At least partly the business stakeholders are drawn into the IT stakeholder's role and must go from their more familiar, informal way of looking at their working situation to a less familiar and more formal approach.

Below we suggest a method that professes to model the social layer. It rests on the assumption that sign processing actors (or stakeholders) are unquestionably to be classified as Information Systems, while IS's are information systems from some perspectives, but also can be viewed as affordances for actors, that are acting in and between organizations, that also can be looked at as actors, for which IS's are affordances.

Since the goal is actionable insight for business stakeholders, we keep our exposition of the method short. In section two we present what we, the developers, could communicate with the (business) project leader who is supposed to be familiar with ontology charts. In section three we describe the KiF-diagram we use with the help of the Cynefin and Sensemaker approach of Snowden. In section four we describe an experimental case study. 


\section{Actualism, Ontology Charts and Interpretation Processes}

Our general model of the social layer for a company starts with the two assumptions of Stampers actualism:

1. No reality without a knowing agent

2. No knowledge of the world without action

From this we construct the interpretation process [agent or state (a), action or effect (b) >- knowledge or response (c)]. The symbol >- is shorthand for the interpretation process that runs from the event of an effect (b) on an agent in a certain state (a) to its resulting conclusion or response (c). The agent can be relatively simple like a tire responding with a flat when undergoing the effect of a nail, leaving room for a causal explanation. But it can also be complex like a whole company responding to market changes with a reorganization plan. The action likewise can be simple like a nail or itself a manifestation of an agent and complex like another business in B to B transactions. The goal can be concrete like a flat tire, or abstract like business objectives. Abstract goals have their reality in that they tend to co-determine the agent's response on an action in instances of interpretation processes. Abstract goals turn a mechanical process of cause and effect into a triadic relation in which actor, action and goal are the corner stones of a complex process.

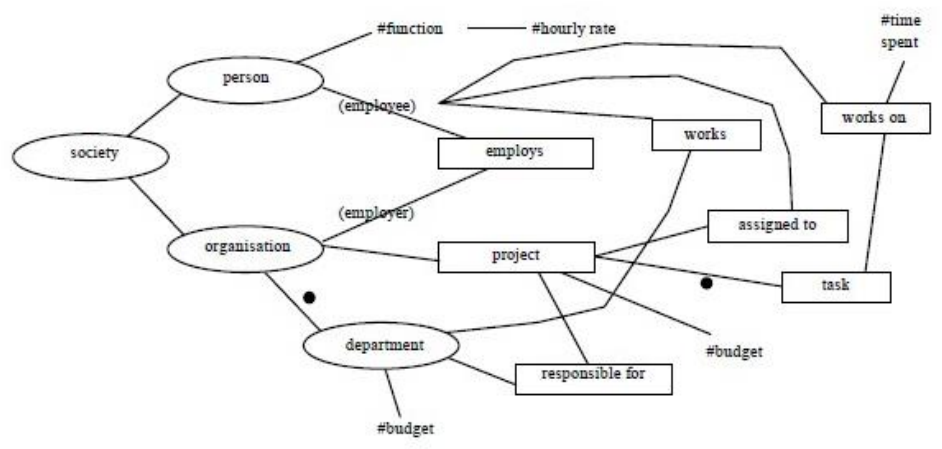

Fig. 1. Ontology chart of project management, cited from Liu 2000, p. 79

For three reasons we will hook our diagrammatic representation of company processes, see below, on the ontology charts of the Semantic Analysis Method (SAM), see fig. 1:

1. Its definition of an agent as a special kind of affordance, that can be defined as something that performs responsible behavior and can be as diverse as an individual, a (part of) organization, a language group, [...].

2. The dependency relation that is assumed and expressed by taking society as the root node of which all other agents are ontological depended specifications. Both points paraphrased from [8]. 
3. The correspondence between these notions in our respective approaches enables the connection of the ontology charts of the semantical level with our diagrams on the social level. This suggests the possibility to connect our proposed technical solution with an existing information system.

In the ontology chart of figure 1 the ontological dependencies are pictured for person, employed by an organization for a department and assigned to a project. In the semantic layer we want to picture the relations between concepts. In the social layer our interest goes to the interactions between the different responsible agents in the execution of their duties. The process definition points the way for a first crude approximation.

Let's say, also as a first introduction of our experimental case below, organization is a company that produces complex tools, materials needed in the operation of the tools and some accessories used in the branch they are in. The department in focus is the sales department populated with sales representatives, each working a rayon. The project aims at improving the sales. To that end we want insight in the training needs of the sales representatives or in terms of the ontology chart the person/department combination. To that end we define the process: Sales Representative (state), Customer (effect) $>$ - Sales (result).

By tracing back to the root node, we find Organization/Company as the node on which the sales department ontologically depends. If we take the Management Team as the responsible agents for this node we get the process: MT, business processes >- Performance. Since the goals of the Sales Department are subservient to the overall business goals, the Sales process is an embedded process. Before we show how this can be captured in a meaningful diagram a few words on the node person and the reason why it appears on the same level as organization.

Suppose that instead of a chart for project management, we want to make an ontology chart for the different organizations a given person contributes to. Besides company, family is an obvious candidate, but also sportive, religious and political organizations are candidates. Each organization has goals the person tries to contribute to in one way or another. And, let's not forget the persons self-interest as a goal pursued. Relevant conflicts of interest may result, and responsibility of agents becomes a subject.

\section{The Cynefin Framework, KiF-diagrams and Sensemaker}

The Cynefin Framework, see fig. 2 left, originates with Kurz and Snowden. The framework offers five contexts in which persons that must make decisions can find themselves to be. It is used primarily "[...] to consider the dynamics of situations, decisions, perspectives, conflicts, and changes in order to come to a consensus for decision-making under uncertainty." It does not aim to categorize a situation but assists in making sense by sharing perceptions [9].

The main division is between ordered (right) and unordered (left) domains. Each domain is characterized according to the cause - effect relationships as they appear to the actors. 

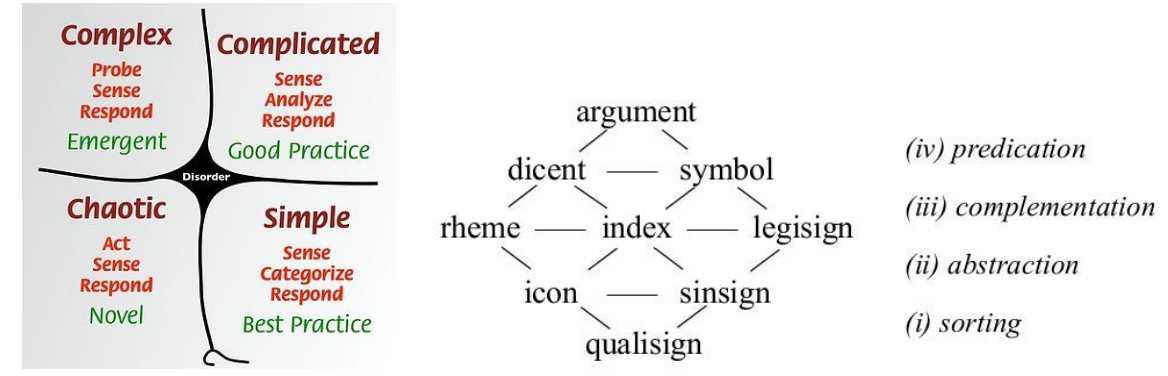

Fig. 2. Left the Cynefin framework with the fifth domain disorder in the center. Next to it the KiF-diagram with the Peircean semiotic terms for the sign aspects on the nodes of the dependency structure that analytically describes the moments each realized interpretation process must pass. Right the four stages in that process. The four square Cynefin domains correspond to the four nodes on the corners: Chaotic - qualisign, Simple - legisign or type, Complex - Rheme or term, Complicated - argument. Disorder corresponds to the index position, for KiF the locus of subprocesses needed to reach a given conclusion. KiF describes interpretation processes, Cynefin indicates the domains in which they may fail and facilitates with the help of Sensemaker the search for solutions.

- Simple or obvious: In this domain the relation between cause and effect is known. The cause we sense is familiar. ${ }^{1}$ We can categorize the cause and respond according to the habit or well-structured set of habits it activates.

- Complicated: In the complicated domain the relation between cause and effect may not be fully known or known only by a small group of people. We must analyze the cause we sense in order to be able to arrange a set of habits for a suitable response.

- Complex: In this domain there are cause-effect relations, but we have insufficient actionable insight in their relationships. The contingencies of history and unintended consequences of previous actions place us in this domain. We must probe the cause from the different perspectives of the involved stake holders and eventually readjust our habits or device new ones for our responses. Safe to fail strategies are advisable.

- Chaotic: Disaster happens. The best course of action is to start acting in order to stabilize the situation, sense the consequences and decide further along the way.

- Disorder: This is the domain of conflict between the different stakeholders about what domain we are in with a given cause. Organizational transformations may suffer from neglect for the deep roots of this domain, it is the locus of cognitive dissonance and a source for conflict about the strategy to follow. ${ }^{2}$

The Cynefin framework facilitates and stimulates dynamical interpretations. A (part of an) organization firmly located in the Simple domain, for instance, may slide into Chaotic if a novelty is recognized not at all, to late or met with a strategy pertaining to a wrong domain. But it does not have a well-defined notion of interpretation processes. The isomorphic KiF-model, first introduced by Farkas and Sarbo [10], contributes one.

1 It is a legisign, alternatively called type or famisign, in Peirce's semiotic terminology.

2 Paraphrased from [9] 
In fig. 2 the KiF-processing scheme is represented with the Peircean semiotic terms that indicate the factors involved in reaching a conclusion (goal) on a sign (effect) that offers itself for interpretation to an interpreting system (state). For details the reader is referred to [11] here we only mention some points relevant for our experimental case.

First, it is important to remark that the KiF-diagram gives an analytical reconstruction of interpretation processes in a dependency structure, it indicates what is involved and does not provide a mechanical bottom up procedure: we suppose the input - output relation to be on a line orthogonal to the index position. Next, we explicate the state and effect below the qualisign position and the goal of the process above the argument position, finally we start to fill in the diagram like a sudoku, see fig. 3 right for an example of the first approximation.

Explications of Cynefin as a rule start in media res with the domain obvious or what is first for us in the order of knowledge. $\mathrm{KiF}$ in contradistinction starts with the first in the order of nature from a cosmic, evolutionary perspective, i.e. the primordial soup (Chaos in Cynefin). ${ }^{3}$ Semiotically the primordial soup is the confluence of all qualities regarded as aspects of signs present to the mind for a process of interpretation that ends with a response. In actual practice habits take a shortcut to a response, the semiotic analysis explicates what we assume to be involved in those habits of interpretation.

Applying the semiotic notions to Cynefin we get a reconstruction of interpretation processes in the most general terms. At the event of an interaction, the state of the agent and the effect are sorted out of the primordial soup. If both fail to be abstracted we are in a chaotic state in which the only course for action is to act, sense and respond without any guarantee. If only the state succeeds we are in a complex situation since there is no best practice (legisign, type) that fits the token (sinsign) we are confronted with. We must probe and sense to familiarize with the effect before an adequate response is possible. If the effect can be abstracted into a type, but the novelty does not fit in with the state (Rheme, also term), the state fails in the complementation state and a suitable response is not found. Again, we find ourselves in the complex domain, with the risk of sliding into chaos. Getting an expert might prove this to be a complicated case, enabling: sense, analyze and respond.

If both succeed and can be complemented, without the emergence of disorder (index), and an adequate response results, we have a best practice and reside in the domain simple. If both can be abstracted but disorder prevents an adequate response we are in the complicated domain. We must sense and analyze before we respond. This is where Sensemaker fits in.

Quantitative data are excellent means to generalize and categorize, but weak in finding the dynamics in individual cases. Qualitative data, like narratives and suggestions, derived from stakeholders are good probes of individual cases. SenseMaker combines quantitative with qualitative data for analytical purposes. Since the reader will be familiar with quantitative data, we concentrate on Sensemakers dealing with narratives. A typical question for Sensemaker is to pose a subject, ask people to tell their story and have them signify the stories with the help of Triads, see for an example fig. 4. the left

3 Cf Aristotle, Analytica Posteriora, 72a. This is what makes Cynefin far superior to the semiotic KiF-framework in explanations for a wider audience of what we do. 
Triad. A Triad is a triangular shape with at each corner a term. The trick is to find three terms that are related and allow for graduation, as in our example. The question posed was: what are your training needs? The score indicates in what amount product knowledge, sales skills and market knowledge are whished for in a training. Of course, in itself the scores on Triads can mean almost anything. But, since the scores are connected to the narratives in the database, by making selections on the scores, the accompanying narratives are retrieved providing the experiences behind the scores, if whished together with gathered qualitative data. Thus, offering handles for interpretation of the scores on the Triads and by that supporting policy making and detecting weak signals.

Use of the Triads in KiF-diagrams differs from their use together with Cynefin. Used together with $\mathrm{KiF}$, the triads must be distributed over the four domains, question and terms on the corners must fit the domain and we ask to provide reasons for the score given. The sign definition, a sign is something (monadic), that presents some object (dyadic relation sign-object), to an interpreting thought (triadic relation of sign, object, interpretant) delivers the format for the terms to choose on the corners, see [12] for the Peircean background. If disorder is an intrinsic feat of the research domain, the Cynefin approach is suited to find weak signals and patterns. If, like in an organization, it is feasible and desirable to resolve disorder, the KiF-diagrams offer a uniform model for all processes and a means to order and distinguish them in dependency structures.

\section{$4 \quad$ An Experimental Business Case}

At some point Nieuwenhuijsen, a trainer in social and commercial skills and interim manager, was asked by a company, vending highly technical tools, to improve the sales of the sales representatives, results deteriorating over time, markets being lost. With Van Breemen he decided that this was a good opportunity to probe the possibilities of the Sensemaking approach for an assessment of the training needs.

The goal of this paper is to propose a diagrammatic method for the social layer, not to analyze a case. For that reason, we will use case data only where it serves the exposition. The actual order of development was: first the left diagram of fig. 3, next the Trikons ${ }^{4}$ left in fig. 4 . The information we got from the sales representatives set us to diagram the remaining two figures for consultation of the MT. Next time we probably will try to follow the order suggested here.

We start with a diagram of the responsible root agent and define the process from the perspective of the goal pursued: MT, events >- sales results, see fig. 3 right. Ontologically dependent agents are embedded processes that reside in the index position. A sale is an exchange of goods and/or services in exchange for money. The company has a Technical Department (TD) in which the tools sold are prepared for sale and adapted to the house style. Our focus is on the Sales Representatives (SR). We indicate both sub-processes as primordial soups with the agent tag below. Regarding each other the processes are parallel processes. Goal and effect are determined in relation to each other

4 Trikons are a special case of Triads because they must adhere to the categorical rules of Peircean semiotics. Space forbids going into details. 
and must fall within the goal of the root process. For feed back to the MT we added marketing, and TD (a second time, as a sub-process of SR).

The left diagram of fig 3. presents the sales process after the state, effect and goal are abstracted from the primordial soup. At the semantical level our interest goes to concepts and their relations. That being covered by the ontology charts (and similar techniques of other approaches), the social layer can be reserved for an interest in the social fabric, i.e. the attitudes that co-determine the outcome of interactions, the characteristics of the agents, their set goals and the characteristics of interactions between agents. In the SR, Customer>- Sales process the domain Simple (legisign position) is reserved for the effect, the other domains pertain to the state. 5 In the mirror process Customer, SR >- acquiring production tools, Simple would be reserved for SR. The reason being that the only thing we can do in any concrete interaction is trying to categorize the effect as good as possible, which includes the possibility of a modification of the self-categorization of the effect and adjust our state. In the Chaos domain (Qualisign) we ask for attitudes that steers the interpretation process of the agent in a state, in Complex we ask for the characteristics that determine the relation of the state to the effect, and in Complicated we try to find out characteristics of the interactions.

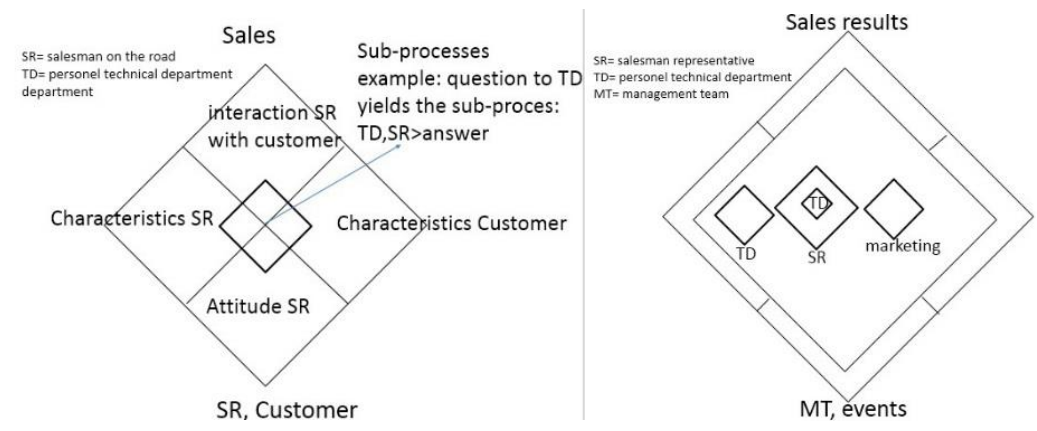

Fig. 3. Left we zoom in on the process [sales representative, customer >- sales]. Right we explicate the sub-processes in the index position from the perspective of sales results.

With this general scheme ready we started to design the Trikons, see fig. 4 left. Experience with interim management and training did help a lot, besides the SWOT analysis that had been made. The urgency behind the innovation process determined our question in the Chaos (Qualisign) domain. We asked for the individual perceptions of the business attitude. Is it a sense of survival, profit or growth that reigns the atmosphere? In the Complex (Rheme) domain we probed the desired kind of training as perceived by the SR: product knowledge, market knowledge or sales skills? In Simple (Legisign) we wanted to find out the customers' needs. In what degree do they need technical

5 If the same process had to be used as a marketing research tool, the domain Complicated would have been devoted to the interaction between SR and customer, but here the focus is on the relations of the SR personnel within the company. 
solutions, product service combinations or best price and delivery conditions? In Complicated (argument) finally our interest went to the social organization of work. Do the SR work on their own, in a hierarchical structure or networked?
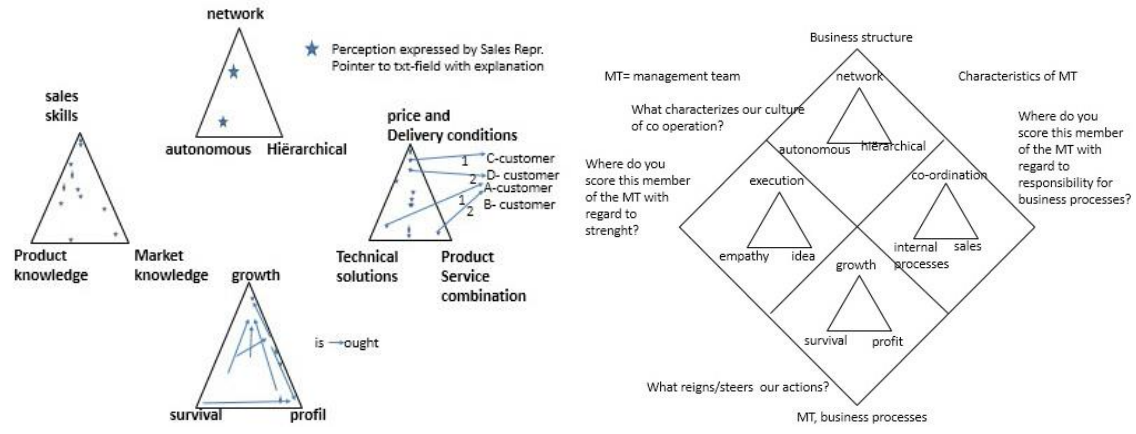

Fig. 4. Left scores of sales representatives for their perceived position on the Trikons. The dots are pointers to texts with explanations for the scores. Right the Trikons we asked the management team members to score themselves on, without the scores.

In two days we interviewed all SR for an hour each. Van Breemen asked the SR to score on the Trikons after that Nieuwenhuijsen had an unstructured, in debt conversation about the reasons for the scores, while Van Breemen acted as scribe. In fig. 4. only part of the scores is given. Analyzing the results of the conversation our conclusion is that we have a promising tool for the MT member responsible for guidance of SR personnel. Four types of issues surfaced.

First, we found strict individual issues to be handled on a personal base: one SR, for instance, felt very insecure, but he proved to be very helpful for SR's with technical questions they didn't get answered by the Technical Department out of lack of time and started calling him for advice. Eventually he went to TD.

Second, on group level, we found issues that ask for a meeting to straighten out the diversity of opinion as well on social as on business issues. Look for example at the diversity of opinions at the attitude question. Such issues ought to be policy driven.

Third, the responsible manager of the SR's, can use this method for progress interviews with personnel to check goals, e.g. in a next interview concerning the trainings needs question: What did you learn? What is your current score on the Trikon? Why?

Fourth, we found structural issues. The SR's fell apart in three groups on the question "Who is your responsible manager?" Sometimes SR's had to call their manager during price negotiation. The bottom price differed, giving rise to uneasiness since part of the wages consisted in a percentage of turn over. This instigated us to design fig. 3 right and fig. 4 right for discussion with MT about business processes and responsibility. 


\section{Conclusion}

In order to actually facilitate actionable insight into the human interplay among signbased world and digital technologies as part of processes of organizational transformation in and between organizations we have to facilitate horizontal alignment at the social layer level and we have to facilitate vertical alignment between the social and the semantic layer. For the social layer alignment issues, we propose our narrative sense making approach. For the vertical alignment issues in our opinion the most promising way to proceed is to systematically work out the connections between our approach for the social layer and the ontology charts of the semantic layer. The pragmatic layer in between consists in the interactions between all those involved in the transformation process.

\section{References}

1. Hoppenbrouwers, S.: Inaugural address, 2013. https://www.han.nl/onderzoek/mensen/stijnhoppenbrouwers/_attachments/intreerede_hoppenbrouwers_2013.pdf. Last accessed $13 / 04 / 2018$

2. Gazendam, H., Liu, K.: The evolution of organizational semiotics; a brief review of the contribution of Ronald Stamper. In: J. F. K. Liu (Ed.), Studies in organisational semiotics. Kluwer Academic Publishers, Dordrecht, 2005.

3. Liu, S., Li, W, Liu, K.: Assessing Pragmatic Interoperability of Information Systems from a Semiotic Perspective. In: 15th International Conference on Informatics and Semiotics in Organisation, ICISO (2014). In: Editors, Liu, K. et al. 15th International Conference on Informatics and Semiotics in Organisations (ICISO), May 2014, Shanghai, China. Springer, IFIP Advances in Information and Communication Technology, AICT-426, pp.32-41, 2014, Service Science and Knowledge Innovation, 2014.

4. Liu, S., Li, W, Liu, K.: Assessing Pragmatic Interoperability for Process Alignment in Collaborative Working Environment. In: Editor, K. Liu et al.: ICISO 2015, IFIP AICT 449, pp. 60-69, 2015., pp. 1-13. Springer, Heidelberg 2016.

5. Weaver, W.: The mathematical theory of communication. In Shannon C.: Some Recent Contributions to the Mathematical Theory of Communication. The University of Illinois Press, (1949) 1998.

6. Goldkuhl, G., Ágerfalk,P. J.: Actability; a way to understand Information Systems Pragmatics. In: Editors, Liu, K., Clarke, R.J., Andersen, P.B., Stamper, R.K.: Coordination and Communication Using Signs; Studies in Organisational Semiotics, pp. 85-115. Springer 2002

7. Oppl S., Hoppenbrouwers S.: Scaffolding Stakeholder-Centric Enterprise Model Articulation. In: Horkoff J., Jeusfeld M., Persson A. (eds) The Practice of Enterprise Modeling. PoEM. Lecture Notes in Business Information Processing, vol 267. Springer, Cham, 2016

8. Bonacin, R., Baranauskas, M. C. C., \& Liu, K.: From Ontology Charts to Class Diagrams: Semantic Analysis Aiding Systems Design. pp. 389-395 In: ICEIS (3) 2004.

9. Kurtz, C.F., Snowden, D.J.: The new dynamics of strategy: Sense-making in a complex and complicated World. IBM Systems Journal 42(3), 462-483, 2003.

10. Farkas, J.I, Sarbo J.J.: A Logical Ontology In: Working with Conceptual Structures: Contributions to ICCS'2000, ed. G. Stumme, pages 138-151, Darmstadt (Germany), Shaker Verlag. 2000. Author, F.: Article title. Journal 2(5), 99-110, 2016.

11. Sarbo, J., Farkas, J., Breemen, A.J.J.: van: Knowledge in Formation: A computational 
theory of interpretation. Springer, 2011.

12. Breemen, A.J.J. van: Quality of Service in the Long Tail: Narratives and the Exploitation of Soft Metadata, IFIP AICT426, 15th International Conference on Informatics and Semiotics in Organisations (ICISO2014), eds. K. Liu, S. Gulliver, W. Li, C. Yu, pages 22-31, Shanghai (China), 2014. 